\title{
Mitochondrial Membrane Protein
}

National Cancer Institute

\section{Source}

National Cancer Institute. Mitochondrial Membrane Protein. NCI Thesaurus. Code C20422.

Including many peripheral or integral membrane-associated enzymes and transport proteins, Mitochondrial Membrane Proteins are found associated with the smooth outer coat or the highly invag inated inner membrane (cristae) of the semiautonomous, selfreproducing cytoplasmic organelles that constitute the principal site of oxidative phosphorylation (ATP formation) for cellular energy in eukaryotic cells. 